\title{
Superiorly based perforator plus flap for inguinal defects
}

\author{
Pradeoth Mukundan Korambayil, Prashanth Varkey Ambookan, Vinoth Kumar Dilliraj \\ Department of Plastic Surgery and Burns, Jubilee Institute of Surgery for Hand, Aesthetic and Microsurgery, Jubilee Mission Medical \\ College and Research Institute, Thrissur 680005, Kerala, India.
}

Address for correspondence: Dr. Pradeoth Mukundan Korambayil, Flat No. 102, Nandana, Haritha Gardens, Vadookara, Thrissur 680007, Kerala, India. E-mail: pradeoth@gmail.com

\begin{abstract}
Aim: Inguinal block dissections for metastasis to inguinal lymph nodes and occasionally trauma are always associated with soft tissue loss over the groin region. A clinical study was undertaken to demonstrate the ability to utilize a superiorly-based perforator flap with reliable vascularity and less donor site morbidity to cover defects in the inguinal region. Methods: A prospective study was performed on 7 patients with inguinal soft tissue defects managed in our institution from January 2013 to September 2013. During the study period, a "superiorly-based perforator plus flap" was used for soft tissue coverage over the femoral vessels in the inguinal region. Hyperbaric oxygen therapy was administered postoperatively. The postoperative period, hospital course, and follow-up after radiotherapy was documented in patients with inguinal block dissection. Results: Seven patients presented with soft tissue defects in the inguinal region. Five of the defects were secondary to prior surgery, and 2 were secondary to trauma. A superiorly-based perforator plus flap was performed in all patients. The defect sizes ranged from $9 \mathrm{~cm} \times 4 \mathrm{~cm}$ to $17 \mathrm{~cm} \times 8 \mathrm{~cm}$. The flap dimensions ranged from $12 \mathrm{~cm} \times 5 \mathrm{~cm}$ to $20 \mathrm{~cm} \times 10 \mathrm{~cm}$. No secondary procedures were necessary following surgery. Postoperatively, there was no evidence of partial or total flap loss. No flap revisions were required, and no complications were experienced at either the donor or recipient site following radiotherapy. Patients were followed-up for 10-18 months. Conclusion: Inguinal defects require stable soft tissue coverage to withstand radiotherapy following inguinal block dissection surgery, and are susceptible to wound complications. The superiorly-based perforator plus flap technique is simple, requires little operative time, and is a reliable flap for coverage of the femoral vessels and inguinal region with improved tolerance to postoperative radiotherapy.
\end{abstract}

Key words:

Hyperbaric oxygen therapy, inguinal lymph nodes, soft tissue defect, superiorly-based perforator plus flap

\section{INTRODUCTION}

Inguinal block dissections are commonly performed for skin malignancies of the lower limb and genital regions. Wound complications including skin necrosis, infection, lymphorrhea, and lymphedema are not uncommon

\begin{tabular}{|l|l|}
\multicolumn{2}{|c|}{ Access this article online } \\
\hline Quick Response Code: & Website: \\
\hline & www.parjournal.net \\
\cline { 2 - 2 } & \\
\hline
\end{tabular}

following inguinal block dissections. Occasionally, trauma may also result in a soft tissue defect in these regions. Morbidity can be reduced by providing a bulky, vascularized flap to the inguinal soft tissue defect, which provides coverage to the femoral vessels, obliterates the dead space, and promotes healing. Reconstructive options currently available for soft tissue defects in the inguinal region include the tensor fascia lata (TFL) flap, the anterolateral thigh flap, and the rectus abdominis flap. We present a "superiorly-based perforator plus flap" which provides stable soft tissue coverage over the femoral vessels, reduces the risk of wound dehiscence and lymphatic drainage problems, improves tolerance to radiotherapy, and decreases the incidence of donor site morbidity when compared to other flaps. 


\section{METHODS}

The study was conducted and approved at the Jubilee Institute for Surgery of Hand, Aesthetics and Microsurgery, Jubilee Mission Hospital, Thrissur, India. The patients were enrolled in the Plastic Surgery Department of the Jubilee Mission Hospital. A soft tissue reconstruction with a superiorly-based perforator plus flap was planned and executed after assessment of the inguinal defect. Seven patients with inguinal soft tissue defects were operated on from January 2013 to September 2013. The postoperative period following reconstructive surgery, the hospital course, and follow-up after radiotherapy was noted in patients who had undergone inguinal block dissection.

\section{Anatomy}

The femoral artery gives off the profunda femoris artery branch, which arises $2-5 \mathrm{~cm}$ below the inguinal ligament and further divides into the medial and lateral circumflex femoral arteries. The lateral circumflex femoral artery further divides into the ascending, transverse and descending branches. The ascending lateral circumflex femoral artery travels in a superolateral direction from medial to lateral. These branches provide many musculocutaneous and septocutaneous perforators which supply the skin over the lateral aspect of the thigh. The vascularity of the skin, subcutaneous tissue and TFL flap is based on the excellent longitudinal network of vessels overlying the iliotibial tract formed by anastomoses between branches of the transverse branch of the lateral circumflex femoral and individual branches of the profunda perforators, which emerge along the lateral intermuscular septum [Figure 1].

\section{Surgical technique}

Surgery was performed under general or regional anesthesia with the patient in the supine position. The soft tissue defect following block dissection or debridement was measured, and the flap was planned. A hand-held Doppler was used to identify the location of the perforators at the base of the flap. A superiorly-based flap was planned from the anterolateral aspect of the thigh, with the base of the flap at the lateral end of the inguinal defect, at the level of the greater trochanter of femur. The anterior border of the flap starts at the lateral border of the soft tissue defect. The flap is elevated from distal to proximal in the plane superficial to the TFL, preserving the perforators to the flap from the lateral side. The pivot point lies over the lateral aspect of the base of the flap. The posterior border is planned according to the size of the defect. The medial flap, overlying the soft tissue of the anterior thigh, was elevated superficial to the deep fascia of the thigh. A blunt, careful dissection is performed at the base of the flap to identify as many perforators as possible in these regions. The size of the perforators is assessed intra-operatively. Because most of the perforators travel from medial to lateral, medial and lateral perforators with minimal dissection could be included within the flap, avoiding kinking of the vessels. With adequate medial perforators, some lateral perforators could easily be sacrificed without compromising flap viability. Care is taken to include some branches of the lateral cutaneous nerve of the thigh to the flap. Anterior branches of the lateral cutaneous nerve lying over the fascia should be carefully preserved for this reason. The medial and lateral flaps are transposed in such way to provide coverage to the inguinal defect and easy closure of the donor defect. Flap inset is then completed. Hyperbaric oxygen therapy was administered in the postoperative period for six sessions.

\section{RESULTS}

Five patients presented with malignant tumors involving the inguinal lymph node and 2 patients presented with defects following trauma. Their ages ranged from 8 to 59 years with a mean of 41.7 years. Among 7 patients, 5 were males, and 2 were females. In all cases, a superiorly-based perforator plus flap was performed. The size of the defect ranged from $9 \mathrm{~cm} \times 4 \mathrm{~cm}$ to $17 \mathrm{~cm} \times 8 \mathrm{~cm}$. Flap with

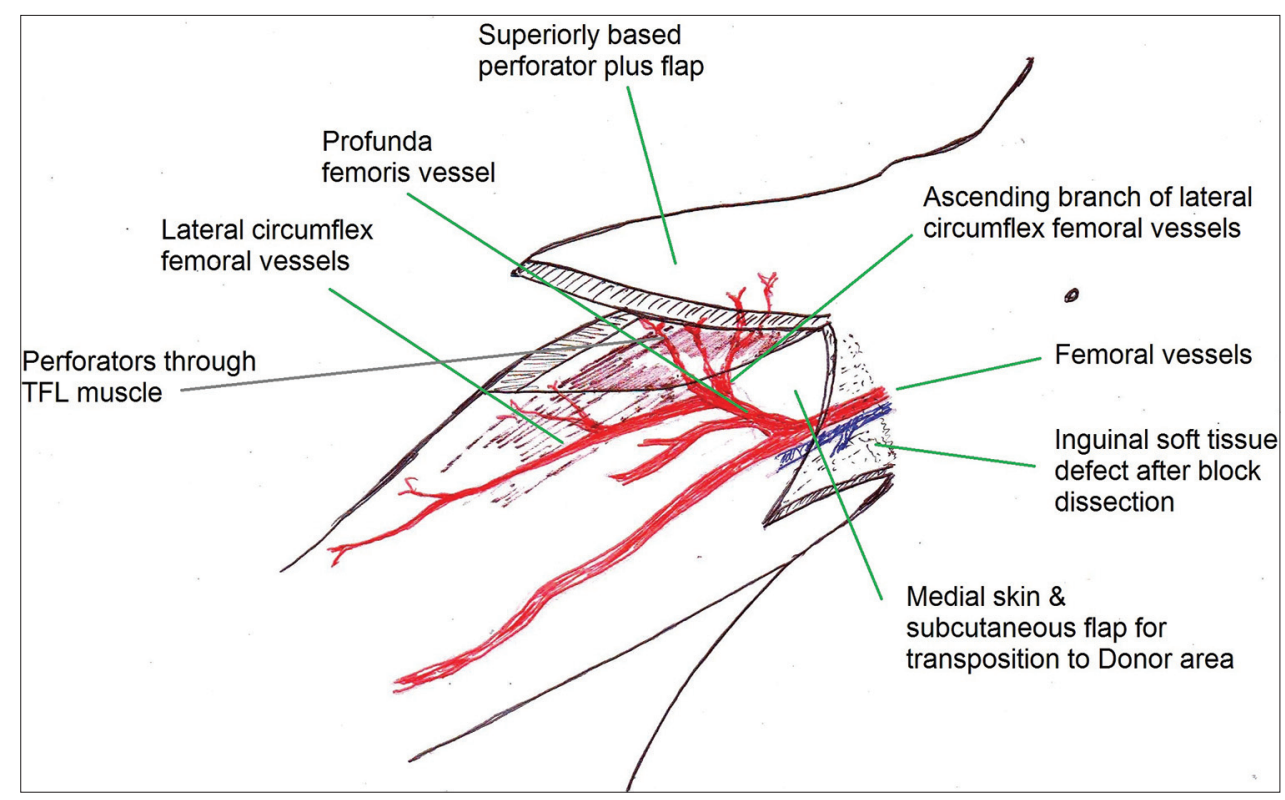

Figure 1: Anatomical description of the superiorly-based perforator plus flap 
dimension ranging from $12 \mathrm{~cm} \times 5 \mathrm{~cm}$ to $20 \mathrm{~cm} \times 10 \mathrm{~cm}$ were created. There was no need for any secondary procedures following surgery. Drains were removed between 4 and 7 days and sutures were removed on the 14th-17th postoperative day. Postoperatively, there was no evidence of either partial or total flap loss. No flaps required revision, and no fat necrosis was noted. There were no flap complications or donor site morbidity following radiotherapy [Table 1]. Patients were followed for 10-18 months after surgery. During this period, there was no recurrence of the tumor in the case of patients who had undergone inguinal block dissection.

Patient with squamous cell carcinoma of the left leg A 58-year-old male patient presented with squamous cell carcinoma of the left leg. Multiple inguinal lymph node were involved and were adherent to each other and to the overlying skin. Wide excision of the primary lesion

Table 1: The patients treated using superiorly based perforator plus flaps for inguinal soft tissue defect

\begin{tabular}{|c|c|c|}
\hline $\begin{array}{l}\text { Age in } \\
\text { years/sex }\end{array}$ & Diagnosis & $\begin{array}{c}\text { Flap dimensions } \\
\text { length } \times \text { width at } \\
\text { base }(\mathrm{cm} \times \mathrm{cm})\end{array}$ \\
\hline 59/male & $\begin{array}{l}\text { Squamous cell carcinoma of } \\
\text { left leg with multiple inguinal LN }\end{array}$ & $20 \times 10$ \\
\hline 8/female & $\begin{array}{l}\text { Soft tissue defect due to trauma } \\
\text { with defect of } 9 \mathrm{~cm} \times 4 \mathrm{~cm} \text { right } \\
\text { groin region }\end{array}$ & $12 \times 5$ \\
\hline $42 /$ male & $\begin{array}{l}\text { Squamous cell carcinoma of left } \\
\text { foot with multiple inguinal LN }\end{array}$ & $18 \times 9$ \\
\hline 48/male & $\begin{array}{l}\text { Squamous cell carcinoma right } \\
\text { foot with fungating inguinal LN }\end{array}$ & $18 \times 10$ \\
\hline $52 /$ male & $\begin{array}{l}\text { Carcinoma penis with right side } \\
\text { inguinal LN }\end{array}$ & $16 \times 8$ \\
\hline 56/female & $\begin{array}{l}\text { Carcinoma ovary with ilio } \\
\text { inguinal LN }\end{array}$ & $19 \times 9$ \\
\hline $27 /$ male & $\begin{array}{l}\text { Soft tissue defect due to trauma } \\
\text { with defect of } 14 \mathrm{~cm} \times 6 \mathrm{~cm} \\
\text { right groin region }\end{array}$ & $15 \times 6$ \\
\hline
\end{tabular}

LN: Lymph nodes and ilio-inguinal block dissection with resection of the involved inguinal skin and soft tissues was performed [Figure 2a]. A plan was made for reconstruction of the [Figure 2b] soft tissue defect over the exposed femoral vessels in the inguinal defect. The superiorly-based perforator plus flap was performed [Figure 2c and d], allowing coverage of the defect and primary closure of the donor site defect. Postoperatively, six sessions of hyperbaric oxygen therapy were administered. The flap healed well [Figure 2e and f]. Regular follow-up was performed. The flap tolerated radiotherapy well.

\section{Patient with fungating right-sided inguinal lymph nodes}

A 48-year-old male patient presented with fungating inguinal lymph nodes on the right side. He had previously undergone surgery for squamous cell carcinoma of the right foot and received radiotherapy to the right inguinal region. A palliative inguinal block dissection was performed [Figure 3a]. The inguinal defect was covered with a superiorly-based perforator plus flap [Figure 3b and c]. Postoperatively, six sessions of hyperbaric oxygen therapy were administered. The flap healed well.

\section{Patient with soft tissue defect over the inguinal region}

An 8-year-old female child was involved in a road traffic accident, resulting in a soft tissue defect over the inguinal region [Figure $4 \mathrm{a}$ and $\mathrm{b}$ ]. The patient was stabilized, and debridement was performed [Figure $4 \mathrm{c}$ ]. The resulting soft tissue defect was covered with a superiorly-based perforator plus flap [Figure 4d and e]. Postoperatively, six sessions of hyperbaric oxygen therapy were administered, and the flap healed well [Figure $4 \mathrm{f}$ and $\mathrm{g}$ ].

\section{DISCUSSION}

Lymph node involvement is an important prognostic marker in primary skin appendage tumors, melanomas

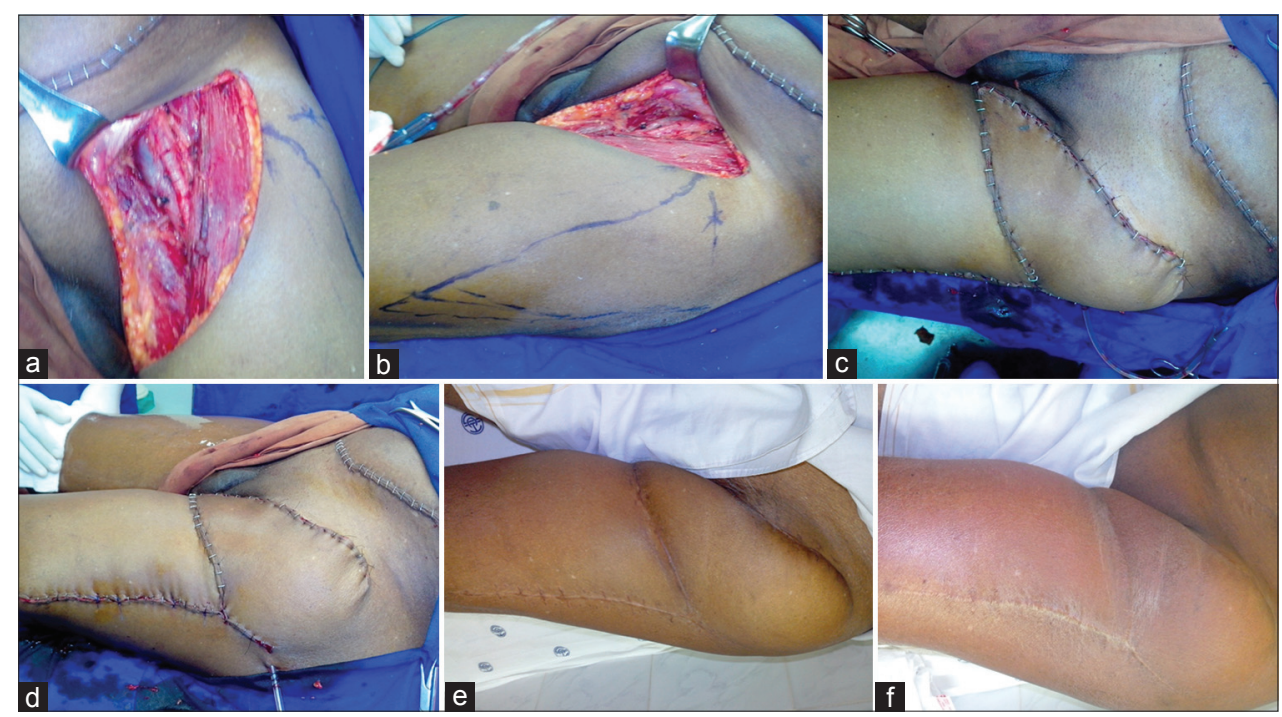

Figure 2: The patient with squamous cell carcinoma of the left leg. (a) Soft tissue defect inguinal region following dissection; (b) planning of superiorlybased perforator plus flap; (c) immediate postoperative view of the superiorly-based perforator plus flap lateral view; (d) immediate postoperative lateral view to show primary closure of donor defect; (e) late postoperative picture after 1 month; (f) late postoperative picture after radiotherapy 
of the genital and anorectal region, and in tumors involving the lower extremities. ${ }^{[1]}$ The clinical presentation of locally advanced primary and nodal disease is not uncommon in India. Surgery may be curative or palliative in such presentations, which requires radical surgery for the primary tumor and en bloc inguinal or ilio-inguinal lymphadenectomy. Inguinal node dissection has been always associated with a high incidence of wound complications. The surgical oncologist has moved from radical dissection to sentinel lymph node dissection to reduce the morbidity due to surgery. Still the role of radical inguinal lymphadenectomy cannot be avoided in certain situations. Potential complications following inguinal block dissection are infection (6-20\%), lymphorrhea (6-40\%), lymphedema (8-69\%) and skin flap necrosis (27-85\%)..$^{2]}$ Removal of the adipofascial layer in a groin dissection damages the subdermal plexus, potentially leading to skin flap necrosis. To reduce complications-related to wound healing, various primary reconstructive procedures such as muscle transposition and myocutaneous flaps are used for groin reconstruction. Many of these patients require adjuvant radiotherapy following surgery. Hence, these patients require stable skin coverage over the operated site for the prevention of tissue edema, fibrosis and complications due to wound

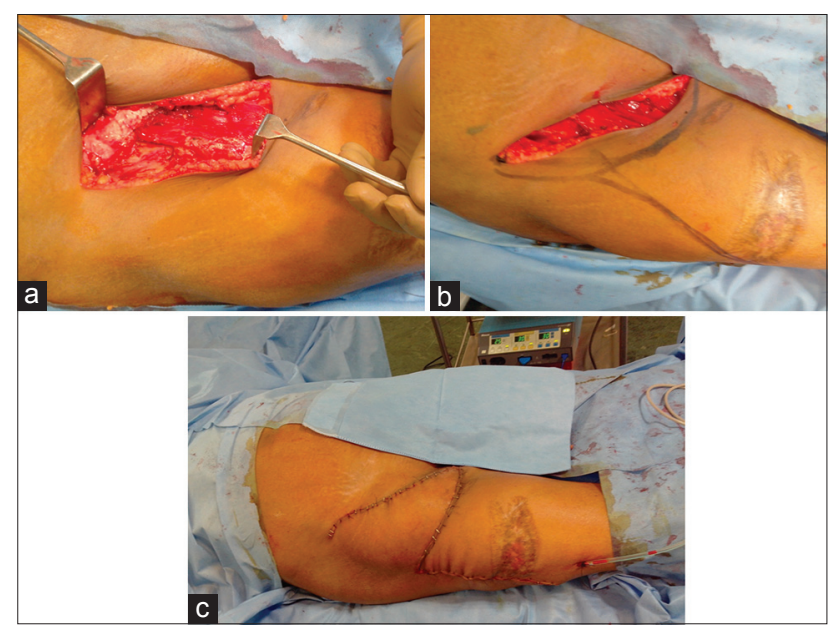

Figure 3: The patient with fungating right sided inguinal lymph nodes. (a) Soft tissue defect inguinal region following dissection; (b) planning of superiorly-based perforator plus flap; (c) immediate postoperative view of the superiorly-based perforator plus flap healing. Primary reconstruction of the groin should, therefore, always be considered for patients undergoing ilio-inguinal node dissection.

Trauma to the inguinal region with soft tissue defects is not uncommon. A high index of suspicion for injuries to the femoral vessels is needed in such cases. With soft tissue defects over the inguinal region, there is always a need for stable soft tissue coverage over the femoral vessels.

Aims of primary reconstruction of the soft tissue defects over the groin region are protection of the femoral vessels, provision of well-vascularized tissue from a distant area, coverage of the dead space in the femoral triangle, a decrease in seroma formation, wound closure without tension, initiation of radiotherapy as early as possible, and a decrease in the length of the hospital stay. ${ }^{[3]}$

Reconstructive options available for coverage of inguinal defects include the random pattern flap, the tensorfascia lata flap, the perforator propeller-type TFL flap, the modified TFL flap, the gracilis and sartorius flaps, the anterolateral thigh flap, the omental flap, the rectus femoris flap and the rectus abdominis flap. Skin grafting is not sufficient for stable coverage over exposed bones, nerves and vessels. Free tissue transfer requires enhanced microsurgical expertise and may overburden patients in critical condition with progressive malignant disease. In such situations, sufficient soft tissue coverage can be achieved by simple and reliable techniques with minimal donor site morbidity.

The TFL flap was first described in 1934 by Wangensteen ${ }^{[4]}$ and was popularized by Nahai et al. ${ }^{[5]}$ for the reconstruction of pressure ulcer defects and for complications following block dissections. Disadvantages of the TFL flap include proximal bulkiness with a thin distal flap, a depressed donor region with an unsightly appearance of the grafted area, and potential loss of stability of the knee due to the sacrifice of fascia lata.

The modified TFL flap includes the muscle with a hatchet shaped incision, which provides adequate mobility of the flap and reduces the dog ear deformity, ensuring closure of the donor area without the need for a skin graft or local flap. Variations in the incision for the flap may not significantly contribute to the reduction of the donor site deformity when the muscle is included in the flap.
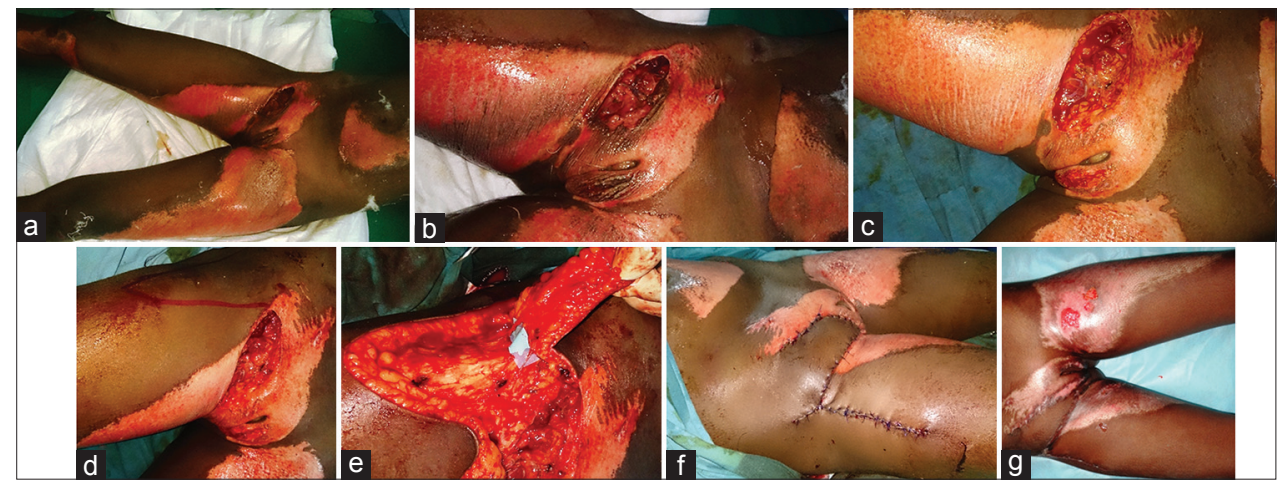

Figure 4: The patient with soft tissue defect over the inguinal region. (a) Soft tissue defect right inguinal region following trauma; (b) closer view of contaminated soft tissue defect groin region; (c) soft tissue defect following debridement; (d) planning of superiorly-based perforator plus flap; (e) identification and preserving the perforators entering the flap; (f) immediate postoperative view of the superiorly-based perforator plus flap; (g) late postoperative picture 
However, in our method, the flap is based on perforators without including the muscle. The requirement for mobilization of the local flap ensures tension-free closure of both the donor and recipient sites.

The perforator TFL flap was first described by Deiler et al. ${ }^{[6]}$ as a free tissue transfer for the reconstruction of Achilles tendon defects. Kimura et al. ${ }^{[7,8]}$ further refined the microdissection technique and described the emergence of septocutaneous perforators between the gluteus medius and TFL muscles. Vegas and Martin-Hervas ${ }^{[9]}$ described the distribution of the branches to the skin from the perforator between the TFL muscle and the gluteus medius and minimus muscles. As a propeller flap, the perforator flap could well be utilized to cover a defect over the inguinal region. The need for microsurgical expertise, the utilization of intramuscular dissection time, the possibility of venous congestion, and the division or noninclusion of the cutaneous nerve within the flap are the disadvantages experienced, while performing this procedure. The superiorly-based perforator plus flap relies on multiple perforators, without sacrifice of the neural component and has a decreased risk of venous congestion when compared to perforator propeller flaps.

Other flaps utilized in the reconstruction of the soft tissue defect over the inguinal region include the anterolateral thigh and vertical rectus abdominis muscle flaps. All these flaps are reliable and provide good soft tissue coverage but at the expense of the sacrifice of a functioning muscle.

After the advent of the angiosomal concept and perforator flaps, skin and subcutaneous tissue-only flaps could be elevated in these regions by preserving the perforators through the area of the TFL. The vascular anatomy of the TFL flap was further studied in detail by Hubmer et al. ${ }^{[10]}$ The author described the blood supply of the TFL as the ascending branch of the lateral circumflex femoral artery with multiple direct septocutaneous and indirect musculocutaneous perforators. Clinically, an additional ultrasound color Doppler is necessary to ensure the perforator point of the flap, because there is always some vessel variation in this region (4-23\%). A perforator flap avoiding the TFL muscle will provide all the advantages of the TFL flap, minimizing the donor site morbidity and other difficulties arising from the bulkiness of the flap.

The literature has demonstrated that hyperbaric oxygen therapy enhances oxygen delivery to peripheral tissues affected by vascular disruption, cytogenic and vasogenic edema, and cellular hypoxia. Tissue edema significantly affects the perforator flaps, wherein the flaps may experience venous congestion. In our institution, we include hyperbaric oxygen therapy in our treatment protocol to reduce postoperative complications related to postsurgical inflammatory events.

In the present study, a superiorly-based perforator plus flap was used to provide stable soft tissue coverage over the femoral vessels, reducing the risk of wound dehiscence and lymphatic drainage problems with minimal donor site morbidity when compared to other flaps. The flap is designed as a random pattern flap based on the subdermal plexus, taking advantage of the rich blood supply; perforators can be added to enhance the viability of the flap. Hence, the flap could be designed with a length: width ratio in the range of $3: 1$. The technique is simple, with lower operating time (approximately 50-60 min), and appears to be a reliable flap for coverage of the femoral vessels and inguinal region with good tolerance to radiotherapy. As a single-stage procedure, the superiorly-based perforator plus flap meets the criteria formulated by Gupta et al., ${ }^{[3]}$ with a reliable blood supply ${ }^{[11,12]}$ and a perforator arterial supply away from the field of resection or radiation. However, assessment of the aesthetic and functional outcomes of the superiorly-based perforator plus flap when compared to other flaps requires additional investigation.

Locally advanced genital malignancies, as well as advanced stage cutaneous melanomas of the lower extremity, are common in Asian populations. Radical surgery for the primary and en bloc inguinal or ilio-inguinal lymphadenectomy is often required in such situations. Posttraumatic soft tissue defects in the inguinal region are not uncommon. To reduce the complications related to wound healing in the groin region and to withstand postoperative radiotherapy, there is a need for a simple, reliable flap in such patients. The superiorly-based perforator plus flap can be successfully used to reconstruct the inguinal region with reliable coverage of the inguinal vessels and early initiation of radiotherapy.

\section{REFERENCES}

I. Murthy V, Gopinath KS. Reconstruction of groin defects following radical inguinal lymphadenectomy: an evidence based review. Indian J Surg Oncol 2012;3:130-8.

2. Swan MC, Furniss D, Cassell OC. Surgical management of metastatic inguinal lymphadenopathy. BMJ 2004;329:1272-6.

3. Gupta AK, Kingsly PM, Jeeth IJ, Dhanraj P. Groin reconstruction after inguinal block dissection. Indian J Urol 2006;22:355-9.

4. Wangensteen $\mathrm{OH}$. Repair of recurrent and difficult hernias and other large defects of the abdominal wall employing the iliotibial tract of fascia lata as a pedicled flap. Surg Gynecol Obstet 1934;59:766-80.

5. Nahai F, Hill L, Hester TR. Experiences with the tensor fascia lata flap. Plast Reconstr Surg 1979;63:788-99.

6. Deiler S, Pfadenhauer A, Widmann J, Stützle H, Kanz KG, Stock W. Tensor fasciae latae perforator flap for reconstruction of composite achilles tendon defects with skin and vascularized fascia. Plast Reconstr Surg 2000;106:342-9.

7. Kimura N. A microdissected thin tensor fasciae latae perforator flap. Plast Reconstr Surg 2002;109:69-77.

8. Kimura N, Saito M, Itoh Y, Sumiya N. Giant combined microdissected thin thigh perforator flap. J Plast Reconstr Aesthet Surg 2006;59: I 325-9.

9. Vegas MR, Martin-Hervas C. The superolateral thigh flap: cadaver and computed tomographic angiography studies with a clinical series. Plast Reconstr Surg 2013;131:310-22.

10. Hubmer MG, Schwaiger N, Windisch G, Feigl G, Koch H, Haas FM, Justich I, Scharnagl E. The vascular anatomy of the tensor fasciae latae perforator flap. Plast Reconstr Surg 2009;124: I8I-9.

II. Cormack GC, Lamberty GH. The vascular territories and the clinical application to the planning of flaps. In: cormack GC, editor. The Arterial Anatomy of Skin Flaps. I ${ }^{\text {st }}$ ed. Edinburgh: churchill Livingstone; 1986. p. 318.

12. Pan WR, Taylor GI. The angiosomes of the thigh and buttock. Plast Reconstr Surg 2009; 123:236-49.

How to cite this article: Korambayil PM, Ambookan PV, Dilliraj VK. Superiorly based perforator plus flap for inguinal defects. Plast Aesthet Res 2014;1:89-93.

Source of Support: Nil, Conflict of Interest: None declared.

Received: 30-06-2014; Accepted: 07-08-2014 